\title{
Utility of Intraoperative Transesophageal Echocardiography in Pulmonary Thromboendarterectomy
}

\author{
Katherine Chaparro, Orozco David Mauricio, Abello Mauricio, Osorio Javier Enrique, \\ Sarquis Tonny Alberto, Barrero David, Hincapie Myriam \\ Department of Anaesthesia, Fundación Clínica Shaio, Bogota, Colombia \\ Email: david.orozco@shaio.org
}

Received 8 January 2014; revised 8 February 2014; accepted 19 February 2014

Copyright (C) 2014 by authors and Scientific Research Publishing Inc.

This work is licensed under the Creative Commons Attribution International License (CC BY).

http://creativecommons.org/licenses/by/4.0/

(c) (i) Open Access

\begin{abstract}
Chronic thromboembolic pulmonary hypertension is one of the few forms of pulmonary hypertension that can be cured surgically, being the pulmonary trhomboendarterectomy (PTE) the therapeutic approach of choice, with survival as high as $\mathbf{7 5 \%}$ to $\mathbf{9 0 \%}$ at 5 years. Our case report focuses on the intraoperative use on the transesophageal echocardiography, which has proven to be of outmost utility in PTE surgery. Two cases of pulmonary thromboendarterectomy are described, in which transesophageal echocardiography was the key factor in the intraoperative management, indicating the initiation time of nitric oxide in case number one and contraindicating the advancement of the pulmonary artery catheter in case number two, due to a mobile thrombus located in the right ventricular outflow tract.
\end{abstract}

\section{Keywords}

Chronic Thromboembolic Pulmonary Hypertension; Pulmonary Thromboendarterectomy; Intraoperative Echocardiography

\section{Introduction}

Chronic thromboembolic pulmonary hypertension is one of the few forms of pulmonary hypertension (PH) that can be cured surgically, being the PTE the therapeutic approach of choice, with survival as high as $75 \%$ to $90 \%$ at 5 years. Our case report focuses on the intraoperative use on the transesophageal echocardiography, which has proven to be of outmost utility in PTE surgery. 


\section{Case 1 (Figure 1)}

A 69-year-old male presented with a history of exertion fatigue that progressed to dyspnea at rest and atrial fibrillation, which was managed electrically. His prior medical history was positive for chronic thromboembolic pulmonary hypertension and renal failure.

A transthoracic echocardiogram was performed demonstrating severely dilated right ventricular cavities, pulmonary hypertension and tricuspid regurgitation; the left ventricular ejection fraction was found normal. The V/Q scan was positive for segmental perfusion defects compromising specially the upper lobes bilaterally. Catheterization revealed both severely elevated pulmonary pressures and pulmonary vascular resistances, 104/38 (61) $\mathrm{mmHg}$ and $1200 \mathrm{dyn} \cdot \mathrm{s} \cdot \mathrm{cm}^{5}$ respectively. He was referred to cardiovascular surgery service for pulmonary thromboendarterectomy.

After the induction of anesthesia, a TEE probe was placed and the following features were specifically evaluated before and after cardiopulmonary bypass:

- Right ventricular ejection fraction (RVEF) and right ventricular dimensions.

- Quantification of tricuspid regurgitation

- Integrity of the atrial septum both with color flow Doppler and bubble looking for the presence of patent foramen ovale.

Our prebypass echocardiographic exam did not differ from the one taken preoperatively, except for a suboclusive plaque located in the right main pulmonary artery.

The pulmonary endarterectomy was performed under 31 minutes of deep hypothermic circulatory arrest during a 168-min pump run. The echocardiographic post-bypass examination showed persistent right ventricular dilation with imminent right ventricular failure and persistence of severe tricuspid regurgitation, so that milrinone and nitric oxide were begun before weaning of cardiopulmonary bypass.

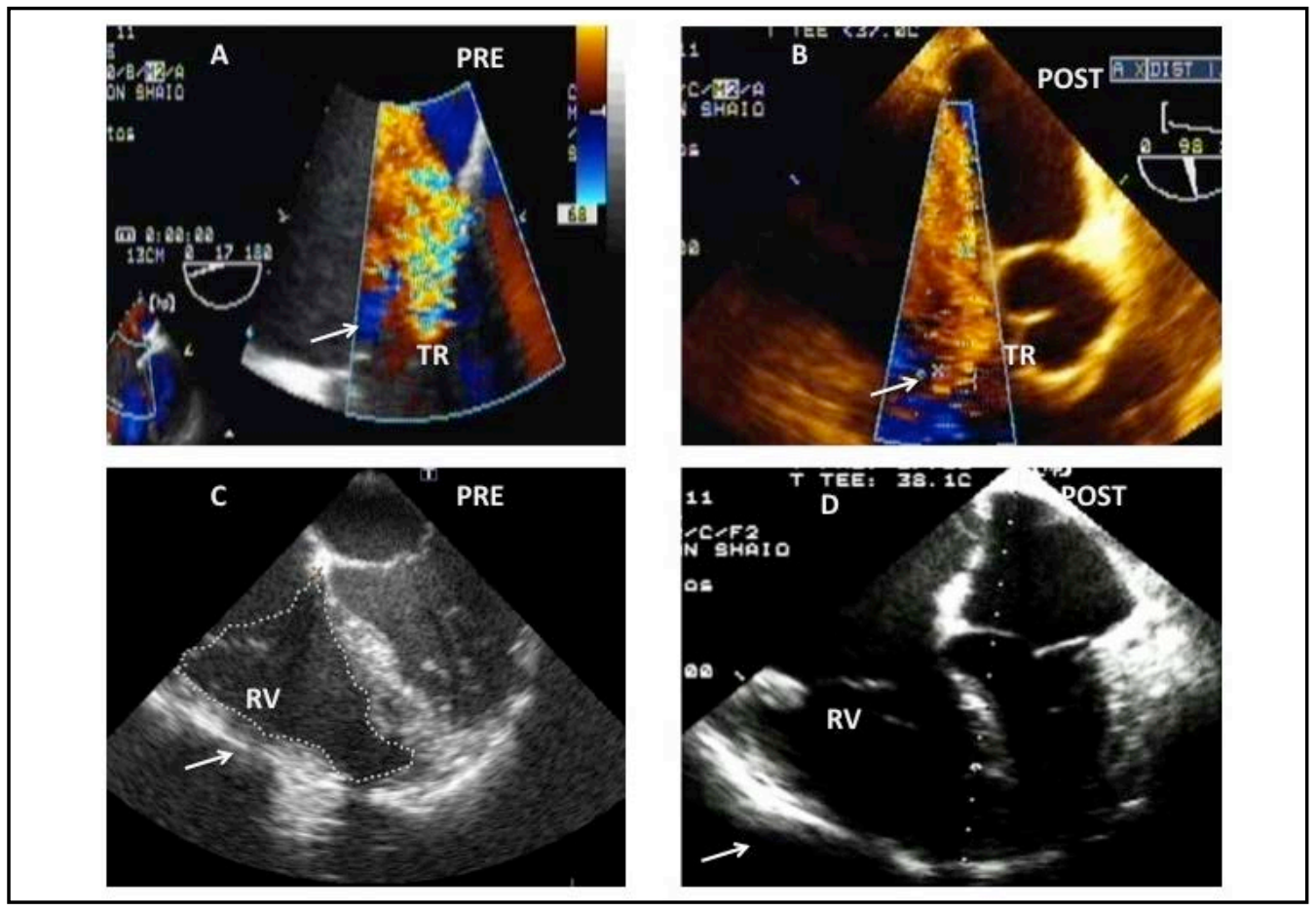

Figure 1. (A) Color flow Doppler interrogation of the Tricuspid valve demonstrating severe regurgitation using the mid esophageal aortic valve short axis view (ME AVSAX) on the prebypass period. The arrow points at the tricuspid regurgitation. (B) Postbypass: the tricuspid regurgitation persists severe. (C) Mid esophageal four chamber view (ME 4CH) with a focus on the right side of the heart demonstrating enlarged right cavities on the prebypass period. (D) Postbypass: Same projection showing severe right ventricular distention, compressing of the left atrial and ventricular cavities. These findings were consistent with imminent right ventricular failure and indicated the inodilator and nitric oxide treatment. The arrow points at the right ventricle. 
The postbypass PVR obtained from the PAC were 589 dyn $\cdot s \cdot \mathrm{cm}^{5}$, which continued to decline to 456 dyn $\cdot \mathrm{s} \cdot \mathrm{cm}^{5}$ on third postoperative day, when the catheter was withdrawn.

The patient was weaned from the aforementioned pulmonary vasodilators plus dobutamine and norepinephrine five day later in the ICU. Later he was switched to bonsentan and sildenafil and continued with his recovery.

\section{Case 2 (Figure 2)}

A 52-year-old female consulted our institution for dyspnea that progressed to NYHA III functional class status, her medical history was positive for hypothyroidism, chronic thromboembolic pulmonary hypertension and a massive pulmonary embolism that required surgical thrombectomy 22 years ago.

The transthoracic echocardiogram revealed severely dilated right ventricular cavities, pulmonary hypertension and normal left ventricular ejection fraction.

An angio CT scan showed a left main pulmonary artery occlusion, compromising elsewhere the left interlobar artery and right lower segmentary arteries.

Catheterization was consistent with severe pulmonary hypertension, 78/33 (51) mmHg and high pulmonary vascular resistances $\left(700 \mathrm{dyn} \cdot \mathrm{s} \cdot \mathrm{cm}^{5}\right)$.

After anesthetic induction, the prebypass echocardiographic exam was done as described in the first case, confirming severe right ventricular cavities dilation and tricuspid regurgitation however, the presence a mobile thrombus located on the right ventricular outflow tract prolapsing to the main pulmonary artery was identified and was not reported in any of the preoperative diagnostic workout.

The possibility of thrombus dislodgment contraindicated the advancement of a pulmonary artery catheter to wedge position.

She underwent surgery under 40 minutes of deep hypothermic circulatory arrest during a 271-min pump run. The echocardiographic post-bypass examination demonstrated adequate resection of the right ventricular outflow mass, improvement in tricuspid insufficiency and right ventricular geometry and function. The pulmonary

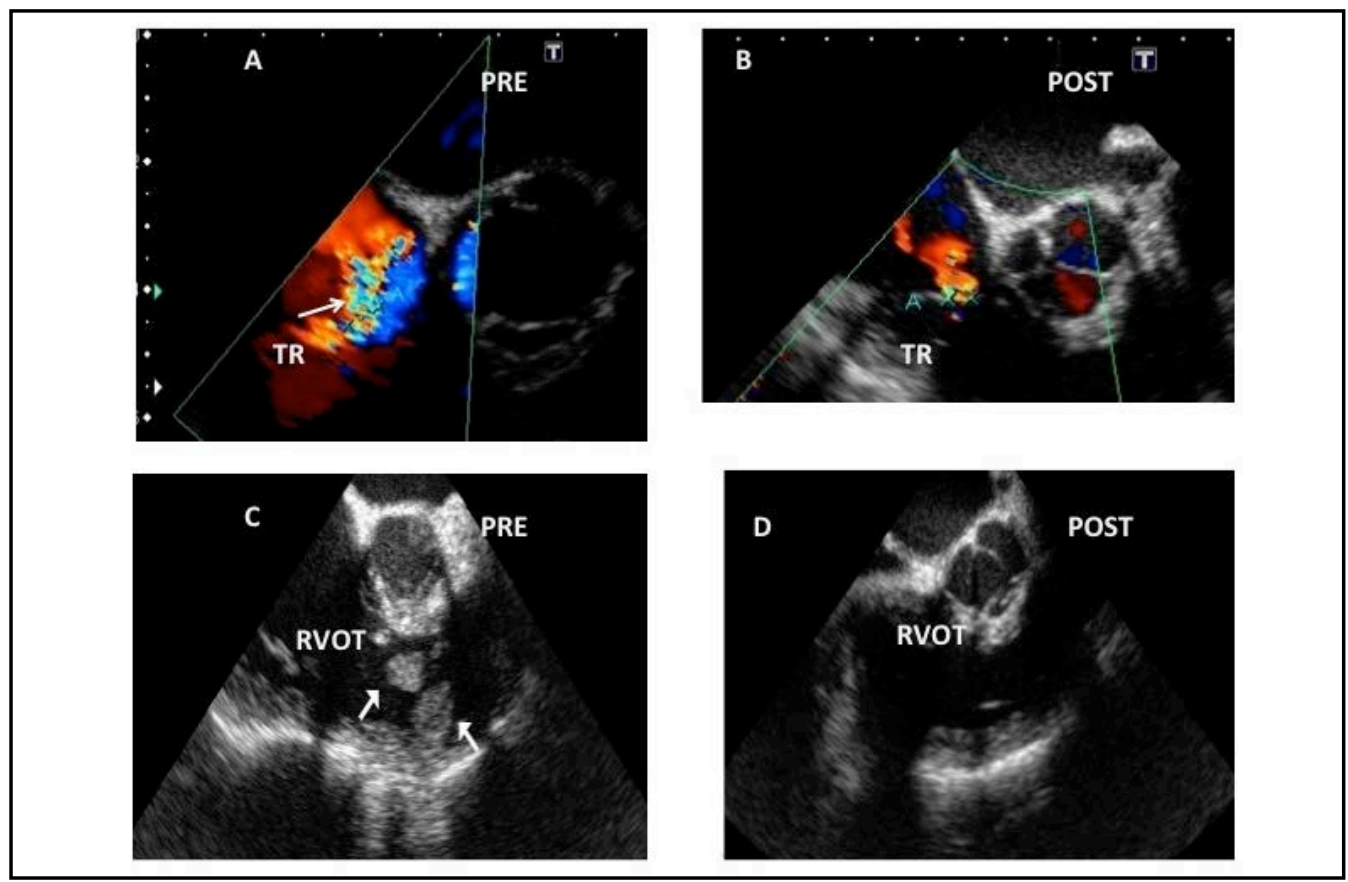

Figure 2. (A) Color flow Doppler interrogation of the Tricuspid valve demonstrating severe regurgitation using the mid esophageal aortic valve short axis view (ME AVSAX) on the prebypass period. The arrow points at the tricuspid regurgitation. (B) Postbypass: the tricuspid regurgitation is mild in the postbypass period consistent with the unloading of the right heart. (C) Mid esophageal inflow-outflow of the right ventricle (ME inflow-outflow RV) showing two mobile masses located in the right ventricular outflow tract. (D) Postbypass: once the adequacy of the removal of the masses was demonstrated, the pulmonary artery catheter was advanced under TEE guidance. 
artery catheter was then advanced under echocardiographic guidance to the pulmonary artery.

The patient was easily withdrawn from cardiopulmonary bypass using low dose dobutamine and norepinephrine. The postbypass TEE reveled reduction of the tricuspid regurgitation and improved right ventricular geometry and function. PVR data derived from PAC was $345 \mathrm{dyn} \cdot \mathrm{s}^{\cdot} \mathrm{cm}^{5}$, thus confirming the TEE findings. She was transferred to the ICU, where she had an uneventful course.

\section{Discussion}

Chronic thromboembolic pulmonary hypertension develops approximately in 1\% to 3.8\% of patients who suffer an acute pulmonary embolism, although its true incidence is unknown [1]. Factors contributing to incomplete clot resolution and progressive growth of embolic material within the pulmonary circulation are obscure and still under debate [2].

CTEPH is one of the few forms of pulmonary hypertension (PH) that can be cured surgically, being the PTE the therapeutic approach of choice, with survival as high as $75 \%$ to $90 \%$ at 5 years [3] [4].

The favorable clinical outcomes depend of appropriated preoperative assessment to diagnose CTEPH and identify the correct surgical patient [5].

Our case report focuses on the intraoperative use on the transesophageal echocardiography, which has proven to be of outmost utility in PTE surgery.

Both cases are clear examples of it. In case number one warning from an imminent right ventricular failure before the weaning from cardiopulmonary bypass, guiding to treatment with inodilators (milrinone) and aggressive right-sided afterload reduction through the use of nitric oxide. Aggressive inotropic support and pulmonary vasodilators is not a frequent practice in PTE surgery, because surgical unloading is the most important factor to reestablish a normal right ventricular function. Therefore the need for inotropic support is usually mild and the use of pulmonary vasodilators rarely justified. However, its use is indicated in residual pulmonary hypertension despite a successful endarterectomy.

The etiology for this residual pulmonary hypertension remains uncertain, but could be attributed to incomplete removal of the occluding material or microvascular vasospasm despite optimal endarterectomy [6]-[8].

Case number two was complicated by the presence of a sessile thrombus on right ventricular outflow tract, which could have been dislodged during the PAC placement. This TEE finding pointed out the best moment for PAC advancement during the rewarming period, once the adequacy of the resection was confirmed.

The main echocardiography aspects to evaluate during PTE surgery, besides the standard intraoperative exam [9] [10], can be summarize as follows [11]:

- Assessment of right ventricular function and dimensions.

- Quantification of tricuspid regurgitation.

- Integrity of the interatrial septum.

The right ventricle is a complex structure, which, unlike the left ventricle, cannot be described by conventional geometric models, making the echocardiographic assessment of this chamber challenging. There are several parameters such as the right Tei index, the systolic excursion of the tricuspid annulus, fractional area change and volumetric methods, that server as estimates of the right ventricular function, however none of them had been validated as the left ventricular ejection fraction. This parameter should be measured before and after the PTE.

The change on the right-sided loading conditions associated with the surgical removal of the obstructive material within the pulmonary circulation causes dramatic improvements on both the function and geometry of the right ventricle, which translates in a measurable decrease of the TR [12].

The final aspect to evaluate is the integrity of the interatrial septum. All defects should be surgically addressed, because they could cause arterial desaturation in a high right-sided pressures scenario and to avoid paradoxical embolism in a patient with a suspected hypercoagulability.

\section{Conclusion}

As a conclusion, we would like to highlight the preponderant role of intraoperative echocardiography in PTE surgery.

\section{References}

[1] Pengo, V., Anthonie, W.A., Lensing, M.D., et al. (2004) Incidence of Chronic Thromboembolic Pulmonary Hyperten- 
sion after Pulmonary Embolism. New England Journal of Medicine, 350, 2257-2264. http://dx.doi.org/10.1056/NEJMoa032274

[2] Gregory, P., Samuel, Z. and Goldhaber (2011) Current Concepts Chronic Thromboembolic Pulmonary Hypertension. New England Journal of Medicine, 364, 351-360. http://dx.doi.org/10.1056/NEJMra0910203

[3] Stuart, W.J., David, P.K. and Naohide, Sakakibara y col. (2003) Pulmonary Endarterectomy: Experience and Lessons Learned in 1500 Cases. The Annals of Thoracic Surgery, 76, 1457-1464. http://dx.doi.org/10.1016/S0003-4975(03)00828-2

[4] Mohammad, R., Tristan, D.Y., et al. (2011) Pulmonary Thromboendarterectomy for Chronic Thromboembolic Pulmonary Hypertension: A Systematic Review. Annals of Thoracic and Cardiovascular Surgery, 17, 435-445. http://dx.doi.org/10.5761/atcs.oa.10.01653

[5] Nick, H.S.K. (2006) Assessment of Operability in Chronic Thromboembolic Pulmonary Hypertension. Proceedings of the American Thoracic Society, 3, 584-588. http://dx.doi.org/10.1513/pats.200605-106LR

[6] Michael, M.M. and Stuart, W.J. (2006) Technical Advances of Pulmonary Endarterectomy for Chronic Thromboembolic Pulmonary Hypertension. Seminars in Thoracic and Cardiovascular Surgery, 18, 243-249. http://dx.doi.org/10.1053/j.semtcvs.2006.09.003

[7] Andrew, R. and Andrew, K. (2008) Pulmonary endarterectomy. Current Opinion in Anaesthesiology, 21, 16-20. http://dx.doi.org/10.1097/ACO.0b013e3282f2bb52

[8] Erica, S., Harish, R., John, G.T. and Augoustides (2011) Recent Advances in Chronic Thromboembolic Pulmonary Hypertension. Journal of Cardiothoracic and Vascular Anesthesia, 25, 744-748. http://dx.doi.org/10.1053/j.jvca.2011.03.182

[9] Practice Guidelines for Perioperative Transesophageal Echocardiography (1996) A report by American Society os Anesthesiologist and the Society of Cardiovascular Anesthesiologist Task Forcé on Transesophageal Echocardiography. Anesthesiology, 84, 986-1006. http://dx.doi.org/10.1097/00000542-199604000-00029

[10] Practice Guidelines for Perioperative Transesophageal Echocardiography (2010) An Updated Report by the American Society of Anesthesiologists and the Society of Cardiovascular Anesthesiologists Task Force on Transesophageal Echocardiography. Anesthesiology, 112, 1084-1096.

[11] Ajit, R. and Ori, B.-Y. (2006) Echocardiography in Chronic Thromboembolic Pulmonary Hypertension. Seminars in Thoracic and Cardiovascular Surgery, 18, 230-235. http://dx.doi.org/10.1053/j.semtcvs.2006.09.006

[12] Andrea, M.D., Giorgio, Z. and Stefano, Ghio y col. (2007) Reverse Right Ventricular Remodeling after Pulmonary Endarterectomy. The Journal of Thoracic and Cardiovascular Surgery, 133, 162-168. http://dx.doi.org/10.1016/j.jtcvs.2006.08.059 